\title{
ucbeci cht.
}

(Die Biffern bejetanen bie Taragrapben.)

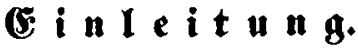

I. Bon Deit Rirdenredt an ith.
A) Rugenteine Begeidnung Deż Gtoffeź
I.

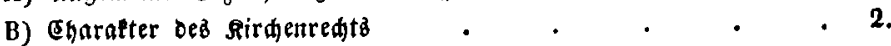
C) Beridieoenheit nad Dem Religionbuetentntnife . , , 3 ,

II. Bon bem sirmenreht ald $\mathfrak{B}$ iffetridnaft.

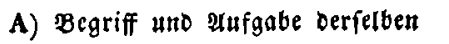
B) Бülfвเwiffenfdyaften

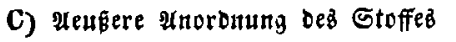
D) \&itterärifhe Sülfzిınitte!

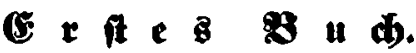

\section{Angemeine CSrund fäbe.}

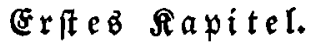

I. Gtiftung ber sirde.

\section{Branblage ber fatholifden Nirdje.}
A) Jefuz हoriftuz
B) Die Upoftel uno igre Beneinden
C) Petrub uno fein Beruf
8.
. . . . 10.

11. Jeftellung Des Begriff Der Rirdie.

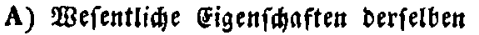
- 11.
B) Berbältniß Der fidjtbaren zur unffđtbaren Rirde $\quad$. 12.
C) Die Rirde in igrer äuperen iroifden Erfdeintung . . 13.

III. Bon Der Riralengewalt . . . . . . . 14.

IV. Non Der Tranbinifiton ber firángenalt . . . . 15.

V. Bon Den Drganen Der Sirdjengenalt.

A) 3ur Berwaltung Der beiligen Sanolungen $\ldots . \quad . \quad$. 16.

B) $\mathfrak{B o n}$ Dem $\mathfrak{e}$ ehrante.
1) Notgronoigfeit Defferben
- 17.
2) Birelide Einfęung einez unfeglbnren sebrantes . . 17a.

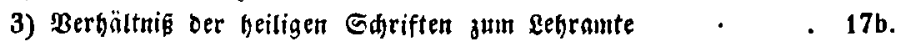
C) Srgane der Sirdenregierung. Sierardjie ber Juriboiction 18.

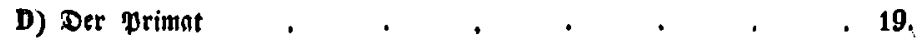




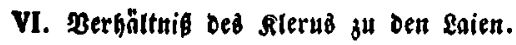
A) Der Rileruä
20.
B) Die Bemeinde
VII. Begenfag ber proteftantifaen 2uffaffung
- 21.

\section{3 weiter $\mathfrak{R}$ apitel.}

(Strunblage ber morgenlánbifáen Sírdłe.

1. Befdidte ber sirdie im Drient.
A) Treıaung von ber abenotänoifden sirde. .
22.
B) Bereinigungåverfude
C) Buftand ber griedifden sirde unter oen Türten
D) Bon oer sirde in Buglano
F) Die unirten Brrieden in polen uno gitthaen
F) Das sönigreid Orriedentand
- $25 \mathrm{~b}$.

I1. Grunblebre ber morgenländifhen Rirdje.
A) Begriff ber Rirdie .
- 26.
B) Bon ber Rirdengewalt
27.
C) Dronung Der Soierardie
111. Angemeine Betradtung
- 28.
- 28 a.

\section{Drittes Rapitel.}

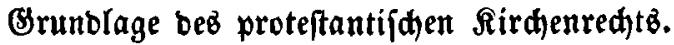

I. Befdidte ber Rirdjentrennung.

A) In Deutfílano.

1) Die Augaburger Eonfefition $\quad . \quad . \quad . \quad . \quad . \quad . \quad 29$.

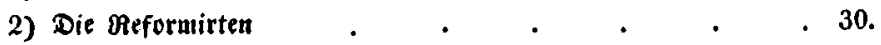

3) Union Der Rutherifiden und Reformirten . . . 30 a.

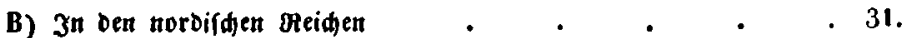

c) 3 in ber Gdweid, frantrcidh und ben Mieberlnnden . . 32.

D) In Englanb, Edjottlant uno Jrlano $\quad . \quad$. $\quad . \quad$. 33.

11. Srunbjüge ber neuen Rirdenverfafiung.

A) Begriff Der Rirde $\quad . \quad$. $\quad . \quad$. $\quad .34$.

B) Bon ber Rirdengewalt.

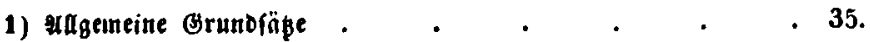

2) formen ber Sirdenverfaffung.

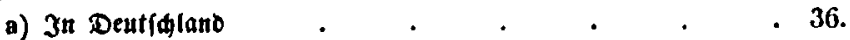

b) $3 \mathfrak{n}$ oen übrigen \&ändern . . . . . . 37 .

3) Neuere Theorien.

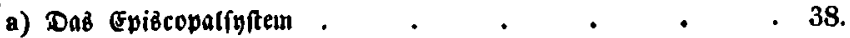

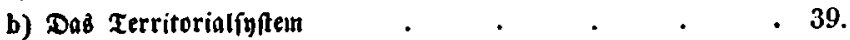

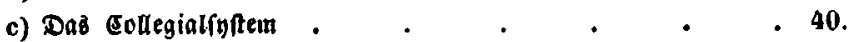

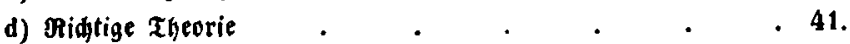

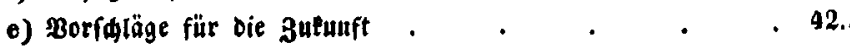


Biertez Rapitel.

₹erhåltniß ocr Rirdje zur Staatżgewalt.

11. Standpunt ber Betradtung

43.

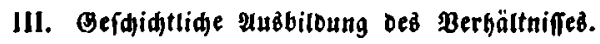
A) Die älteren 3 eiten
B) uebergang zur neueren 3 eit
44.
C) Die falide Theorie ber Squle.
1) Dad ius advocatiae
46.
2) Dab ius cavendi.
a) Bebeutung Deffellen . $\quad . \quad . \quad . \quad 46 \mathrm{a}$
b) Daв ßedit Der Dberaufídt . . . . . . . 46 b.

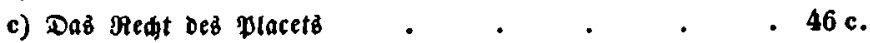
d) Die Mitwirfung bei ber थnftellung Der Rirdenbeanten $.46 \mathrm{~d}$.
e) Die 2apellation ab abusu . . . . . 46 e.

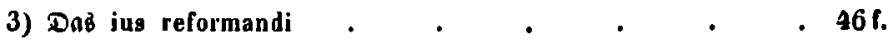
4) Daz Dbereigentbum über ๖az Rirфengut . . . $46 \mathrm{~g}$.
v) Die neueften Ereigniffe $\quad . \quad \ldots \quad . \quad . \quad .47$.

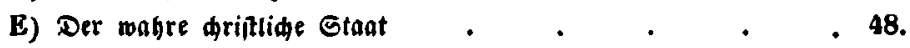

$\mathfrak{F} \mathfrak{u} \mathfrak{n}$ fte $\mathfrak{B} \mathfrak{A} \mathfrak{a}$ itel.

Mon bem $\mathfrak{B e r h d l t n i}$ veridiebener Religionbitheile gegen einanber.

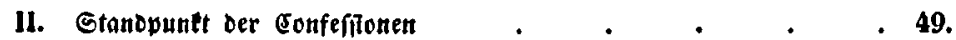

III. Stanopuntt dea Staated.

A) altea Redit $\quad . \quad$. $\quad . \quad$. $\quad . \quad 50$.

B) Brundiäpe dez deutidien Staatzrenta.

1) Heber baz Berbältnif jwifden ben Ratboliten unb proteftanten

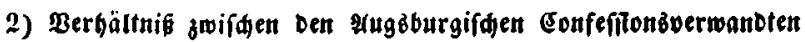
und Den Meforutirten . $\quad . \quad$. $\quad . \quad$. 52.

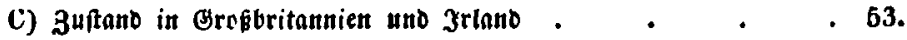

D) 3uftano in ben anderen Reiden . . . . . 54.

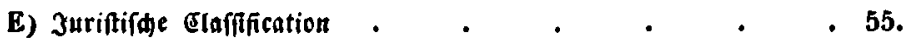

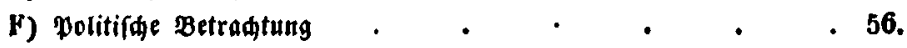

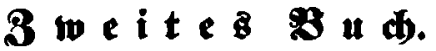

Bon den Quelten bes Sirdjenredfts.

Eriteb $\mathfrak{A} a p i t e l$.

Arthemeine Befdaffenheit berfelben.

II. Quellen bes eatbolificen Rirdenredits.
A) Borínriften Ebrifti uno ber apoftel
- 57.
B) Eoncilienfalüfe
C) Päpftlide Eonftitutionen .
58. 


\section{- XIY -}
D) Eoncorbate unt welttide Befę̧e . $\quad . \quad$. 60
E) Particularredte eingelner Diöcéen $\quad$ - $\quad$. 61 .

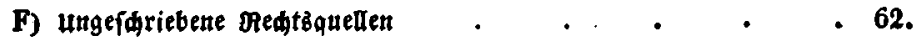

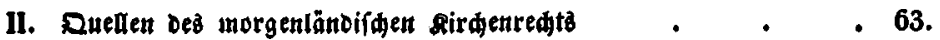
III. Quellen Des proteftantifकen Rirdentredt . . . . 64.

\section{3weitez $\mathfrak{A}$ apitel. \\ Sejdjidte ber Quellent.}

1. 3uftand Des Stirdenredtb bis ins fünfte 3ahrgundert.

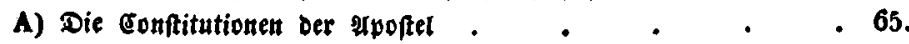

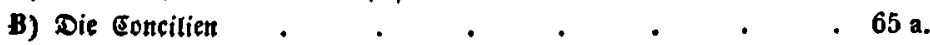

C) Eanontenfaumlungert.

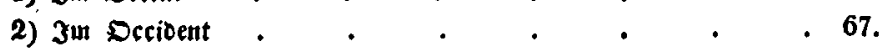

D) Die Gaunlung ber Eandnen ber atpoftel $\quad . \quad . \quad, 68$.

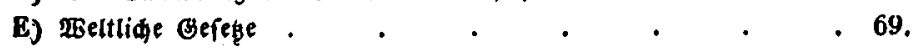

II. Befondere Befdidte des morgentänoifden Rirdentedjtb.

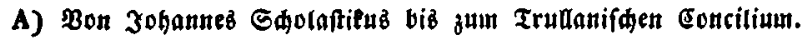

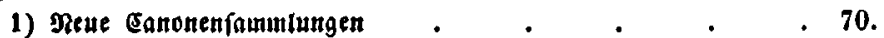

2) Beltide ORedtiqueller.

a) Bewögnlide Gamunlungent Derfelben . $\quad$. $\quad$. 71 .

b) Befondere für die Rirde beftimmte Sammlangent.$\quad$. 72.

3) Bemif́lte Gamulungen . $. \quad . \quad . \quad . \quad .73$.

B) Boun Trullanifhen Eoncilium biß photiub.

1) Bermebrung ber canonenfaumlung . . . . 74.

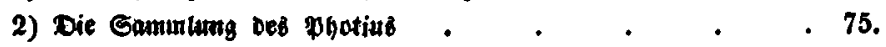

C) Bon Photiuz biz auf bie neuere 3eit.

1) Buftano Deb griedifdent Sirmenredtz.

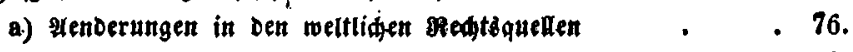

b) Die tirdliden Samulungen . . . . , 76.

c) Eommentarien $\quad . \quad . \quad . \quad . \quad 77$.

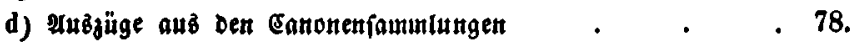

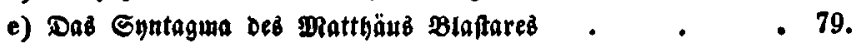

f) Seutiger Buftand $\quad . \quad . \quad . \quad . \quad . \quad . \quad 80$.

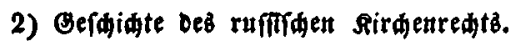

a) $3 \mathfrak{n}$ älteren 3eiten $\quad . \quad$. $\quad . \quad . \quad . \quad .81$.

b) Seutiger 3uftand $\quad . \quad+\quad . \quad \ldots \quad . \quad \cdot 82$.

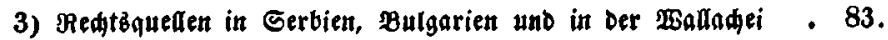

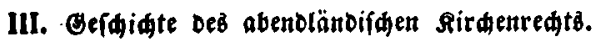

A) Boun fünften biş jum neunten Jabrfundert.

1) Einzelue Reqtiquenten .,$\quad . \quad . \quad . \quad 84$. 
2) Quetlenfaumlutgen.

a) In Italien.

a) Gaumlungen ber Rirdengefépe.$\quad \cdot \quad \cdot \quad .85$.

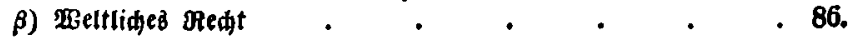

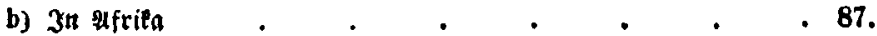

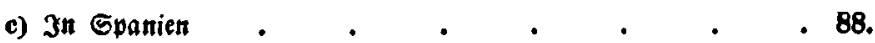

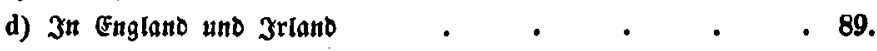

e) $3 \mathfrak{n}$ Gallien uno dem fräntifden Oreidje.

a) Eanonenfaunutungen . $\quad . \quad$. $\quad . \quad 90$.

в) હyftenatifde હamulunget: . . . . . 91.

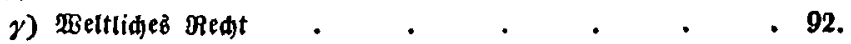

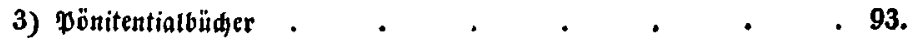

4) Ritual= und Formelbüder . $\quad . \quad$. $\quad . \quad$. 94.

B) Die Samulung Der falfden Decretalen.

1) Brefilite berfelben $\quad . \quad$. $\quad . \quad, \quad, 95$.

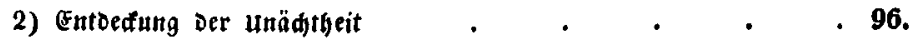

3) Rritifde unterfudungen $\quad . \quad$. $\quad . \quad+\quad . \quad .97$.

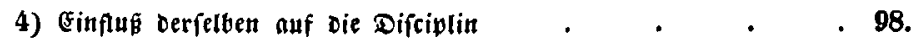

5) Indere Danit verwandte Gammlungen . . . . . 99.

C) Bom zeknten biß̈ zum zwölften Jahrgumbert.

1) Die Sanmmlungen vor Bratian . . . . . 100.

2) Die Samurlungen Ded Bratian and Dez \&aborans.$\quad 101$.

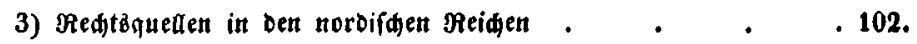

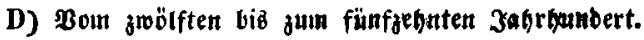

1) Dad Bemeitfame.

a) Die nflgemeinen Eoncilien $\quad . \quad \ldots \quad . \quad . \quad 103$.

b) Reception Der Gaumlung Bratianz auf ben untiverftäten .104.

c) Die Decretnlenfammlungen vor Bregor IX. . . . 105.

d) Die Decretalenfammlungen feit Bregor IX. $\quad . \quad$. 106.

e) Selvitfändige MBerfe über bas canonifde «Redt $\quad$. 107.

2) Befondere $\Re e d t$ tzuellett in bett eingelnen Beident.

a) 疎 Deutidinno, Franlreid, Englano uno ungarn $\quad . \quad 108$.

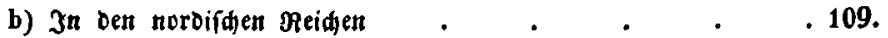

E) Dả fünfzehnte Jabrhundert.

1) Die Eoncilien $\quad . \quad$. $\quad . \quad$. $\quad .110$.

2) Rüdroirtung auf Die eingelnen Ränder . $\quad$. $\quad$. 111.

F) Die orei leg̨ten $\mathfrak{a h h r b u n b e r t e . ~}$

1) 3uftand des entyolifien Rirdjenredta.
a) Dả Eoncilium von Trient

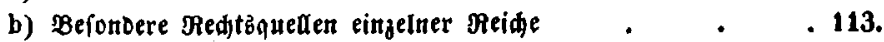
c) Einfug neuer ooctrineller Dreinungen . $\quad . \quad . \quad .114$.
d) Einfluś der franzöififden Revolution . . . . 115.
-) Die neueften Bedfizuetlen . . . . . 116. 
2) Befaidite bes proteftantifaen Rirdenredits.

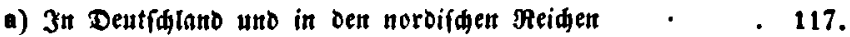

b) 3nt Frantreid, Den Niedertanden, England und Gdottland 118.

Drittez $\mathfrak{A} \mathfrak{a}$ itel.

Bon ben Quellen beż Rírdjenredtż nad ifrem praftífden (Sebrautde.

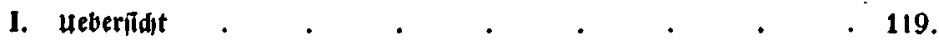

II. Die Gamulungen Deb geltenoen Rirdenredtz.

A) Beftalt Derfelben.

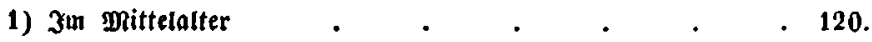

2) Meuere Beränderungen $\quad . \quad$. $\quad . \quad$. 121.

B) Don bein gefegliden Infegen ber Samminngent bez canonifhen Deditz . . . . . . . . . 122.

C) Seutiger Gebraud Deß corpus iuris canonici . . . 123.

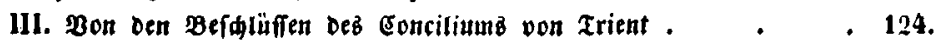

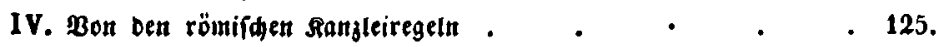

I. Bon Dem Primate.

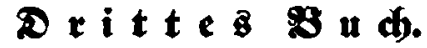

Bon Der $\mathfrak{B e r f a f f u n g}$ Der Rirche.

$$
\text { Erite } \mathfrak{A} a \text { pitel. }
$$

Bom Papfte und beffen Bebúlfen.
A) 3un भagemeinen
126.
B) Redte Deffclben
C) Doctrinelle 2 Inildten über Daffelbe
- 127.
D) Efrenredte bes papfes
- 128.
E) Bon beur Rirdenftante
- 129 .

II. Bon ben Eardinälen.
A) Befdidte Diefer Bürbe .
- 131.
B) beutigez oRedt
$132-132 \mathrm{c}$.

III. Bon Der röunifden Eurie.
A) Päpfltide Regierunga = und Juftidcollegien
- 133.

B) Eongregationen oer Earbinäle .

IV. Bon ben apoftolifden Legaten uno Bicarien.
A) Berbältniffe der älteren 3 eit
135.
B) Berbăltniffe ìn Mittelalter
- 136.
C) seutiges Bedt
- 137.
V. Die Bebörden jur Berbreitung Ded ৫Iaubenz . . . 137 a. 


\section{3weitez $\mathfrak{x a p i t e l}$.}

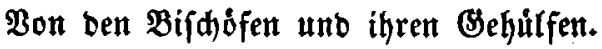

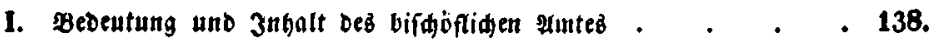

I1. Bort Den Raviteln.

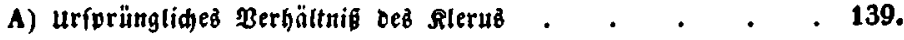

B) Entftehung Dez canonifめen sebenż . . . . . 140.

C) Beränderungen in Mittelalter . . . . . . . . 141.

D) Soeutiged $\mathfrak{R e d t}$.

1) 3ufaumenfegung Der Rapitel $\quad$. . . . . . 142,

2) Oedte Der Rapitel . . . . . . . . . . 143.

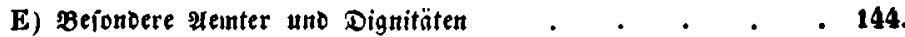

III. Gehülfen unb Stedvertreter Der Bif́b̈fe.

A) Bewöhnlide.

1) Fïr Die beiligen Berridtungen . . . . . . 145.

2) Bebülfen für die äufiere Berwaltung . . . . . $145 \mathrm{a}$.

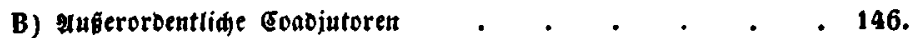

IV. Bon ben $\mathfrak{P}$ farrern.

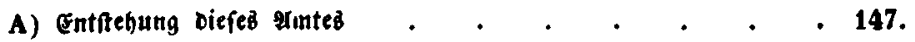

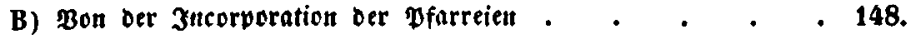

C) Bon ben $\mathfrak{D f a r r e r n}$ und Deren Behülfen nah Dew beutigen Redt 149.

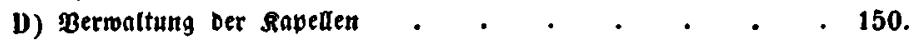

V. Bon ber bifqüfliden હurie . . . . . . . . 151.

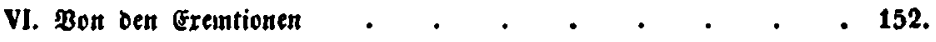

\section{Drittes Sapitel.}

Bon ben Erzbijadfen, Exarden, ஒatriarden unb Primaten.

1. Bon Den Erabifä̈fent.

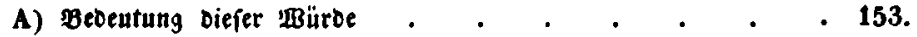

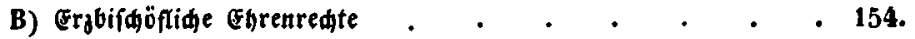

11. Exarden, Patriarmen uno primaten . . . . . 1 . 5.

\section{Bierte Rapitel.}

Bont ben Eoncilien.

I. Einleitung . . . . . . . . . 156 .

II. Bon ben allgemeinen Eoncilien.

A) Einribtung berfelben . . . . . . . . 157.

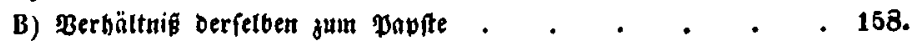

III. Bon Den פational= und provinzialconcilien $\quad . \quad . \quad . \quad$. 159.

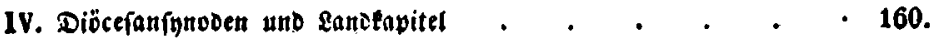

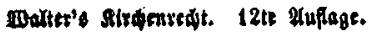




\section{- XVIII -.}

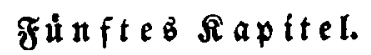

Berfáfung ber morgenlánbifden Rirdje.

Einleitung

I. Berfaffung iut Patriardate.
A) Die Patriarden
B) Die Biføöfe uno übrige Beiftlidteit
162.

II. Die tirdilide Berfaffung in Bußlano.

A) Die heilige Gynode . . . . . . . . . . $\quad .164$.

B) Die Bif́p̈fe uno übrige Geiftidteit . . . . . . 165.

III. Daß Rönigreid Brięenland . . . . . . . . 166.

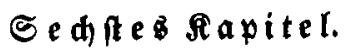

Beiftlide Berfafiung ber proteftantifa)en Ranber.

1. Berfaffung in Deutfidant.
A) \$anbbabung Dez sirdenregiments
167.

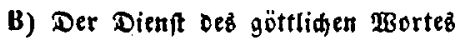
168.

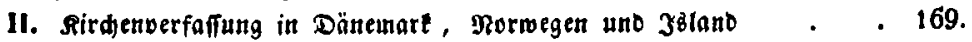
III. Sirdenverfafiung von Sdroeden . . . . . . . . . 170 .
IV. Berfaffung ber englifhen Evižcopaltirae . . . . . 171.
v. Sirdenverfaffung in Benf, frantrei内 uno Edottlano . . . 172.
VI. Rirdenterfafiung it ben Niederlanden . . . . . . 173.

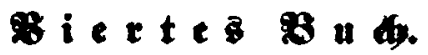

Bon der Berwaltung ocr fittife.

Erftes Sapitel.

Berwaltung ber heiligen Sanblungen.

I. Matur Diefer Berwaltung . . . . . . . . 174.

1I. Ber低iedene Gtufen ber Berwaltung . . . . . . 175.

\section{3 weite $\mathfrak{R} a$ pitel. \\ Berwaltung ber Rehre.}

1. Bon Der Erbaltung ber gebre . . . . . . . 176.

II. Bon oer Berbeitung ber \&effe . . . . . . . . . 177.

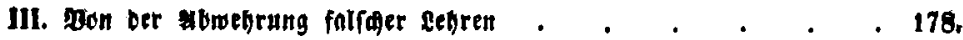




\section{XIX}

\section{Drittes Rapitel.}

1. Bon Der Befeggebung.

Bermaltung per Difciplin.
A) Theorie Derfelben
179.
B) Bon Den Drivilegien uno Dispenjationen . . . . 180.

II. Bon ber geiflidjen Beridtobbarteit.

A) 2unenoung berfelben.

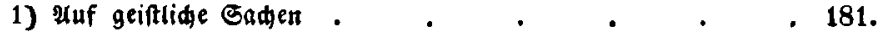

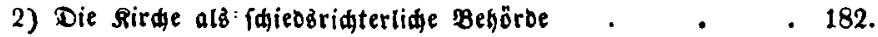

3) Die firde alz frivilegirter Beridtâkand der Beiftiden 183.

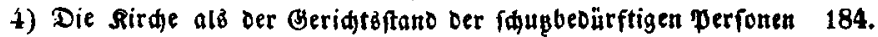

B) Bon ben geifliden Beridgten . . . . . . . . 185.

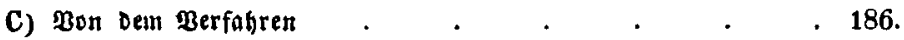

III. Berwaltung Der Dberaufindt $\quad . \quad$. $\quad . \quad . \quad .187$.

IV. Bon Der firdliden Gtrafgemalt.

A) Gegenftände derfelben.

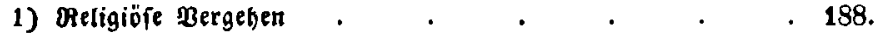

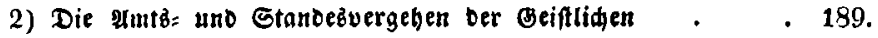

3) Die Rirde ala privilegjeter Geridtäftanto ber B̉eiftliden • 190.

B) Bon Den firqli申en Strafen.

1) Eingelne 2rten.

a) Begen Raien . $\quad . \quad . \quad . \quad . \quad 191$.

b) Oegen Beiftide $\quad$. $\quad$ - . $\quad$. 191 a.

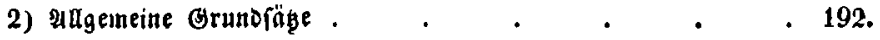

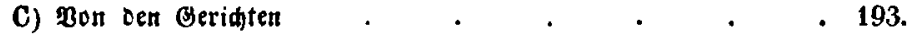

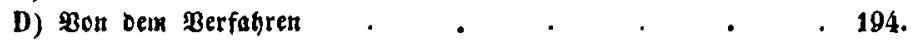

v. Bon beun tird)liden Befteueruagäredr.

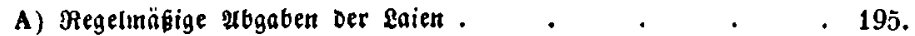
B) Ubgaben bei befonderea Serfären $\quad$. . . . . 196.

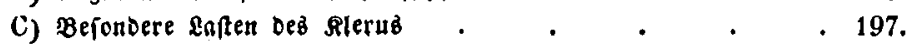
D) Befonbere abgaben ant bent.
1) Zeltere formen $\quad . \quad \ldots \quad . \quad . \quad . \quad 198$.
2) eugaben bei Der Berleihung oer Rirden⿳亠幺⿲丿丨丶
a) Siftorifhe Einleitung $\quad . \quad$. $\quad . \quad$. $\quad$. 199.

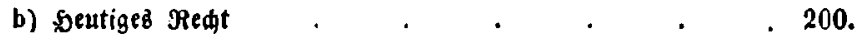

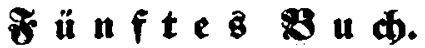

Bon Dem fird)liden-Beamtenmelen. Erftez Rapitel.

Bon ber Ergiehung ber אlerifer.

I. Berbältniffe ber äıteren ३3eit

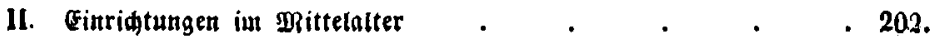

III. Seutiger Z3uftano . $\quad . \quad$. . . . . . 203. 


\section{3 weited $\mathfrak{R} \mathfrak{a}$ itel.}

Bon ber Sroination.

I. Bebeutung ber Droitntion

II. Berfidiebene Etufen ber Drbination.

A) Die Tonfur und die fieven weiben $\quad . \quad$. $\quad . \quad 205$.

B) Unterfajied ber fö̈heren und niederen IBeigen . . . 206.

III. Bon oer Befugni占 ju oroiniren . . . . . . . 207.

IV. Bon der Fäbigleit orbinirt zu werben . . . . . 208.

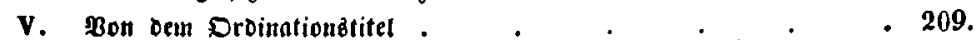

vI. $\mathfrak{B o n}$ Dem $\mathfrak{B e r f a h r e n}$ bei ber Ordination . . . . $\quad .210$.

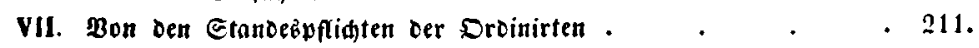

vIII. Bon ber Berpfliatung jun ebetcien \&eber.
A) Siftorifade Einteitung
- 212.
B) Seutigeø̉ $\mathfrak{R}$ edt
C) Rugemeine Bemertungen
- 213.
. 214.
1X. Ragemeine Etanoebredte Der Geiflident . . . . 215.

\section{Dritteb Rapitel. \\ Bon ben Rirdpenámtern in Allgemeinen.}

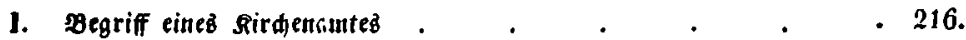

11. Gintheilung ber şirdhentüter . . . . . 217.

11. Erridtung Der Sirdjenn̈uter . . . . . . . 218.

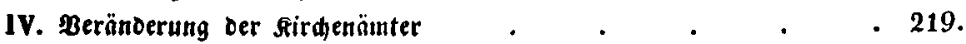

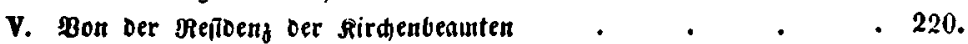

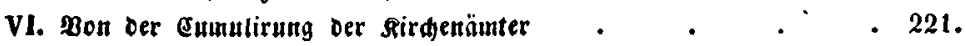

\section{Bierteg Rapitel.}

Bon ber Befę̧ung ber Rírdenámter.

1. ueberint

11. Ratholifde3 Rirdentredt.

A) Befegung Der Biathümer.

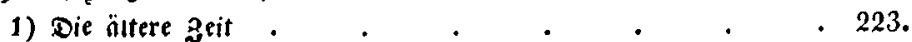

2) 3 uftano in ben germanifden $\Re$ eiden . . . . 224.

3) Hevergang in Die newere form . . . . . . 225.

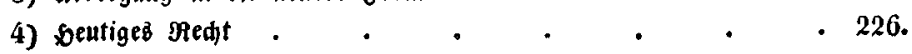

B) Bon Der Wahl Dez Papftez.

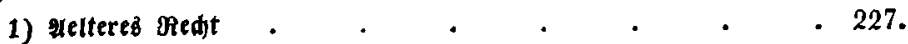

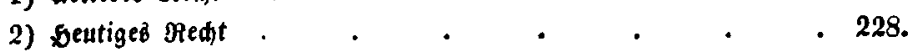

C) Befę̧ung Der übrigen Rirdenämter.

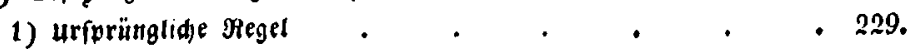


2) Befequng Der Rapitel.

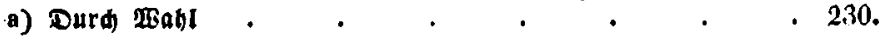

b) Durd päpflide Pantate unt Inwartidafter . . 231.

c) Durd päpftlide Mefervationen . . . . 232.

d) Neutefte Einri由tungen . $\quad . \quad$. $\quad . \quad$. $\quad . \quad$. 233.

3) Einflus Des Datronatredtz.

a) biftorifhe Einleitung . . . . . . . 234.

b) Weutigez Redt . . . . . . . . . . 235.

4) Boషlez Berleihungäredt oritter perfonen . . . 236.

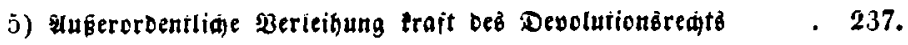

6) Bon oer canonifden Inftitution uno ber Jnveftitur . . 238.

III. Zuftano der morgenländifden Rirde. . . . . . . 239.

IV. 3uftant in ben froteftantifaen gändern . . . . 240.

V. Bemeinfdaftlide (frfordernife $. \quad . \quad . \quad . \quad$. 241.

\section{$\mathfrak{F} \mathfrak{u} \mathfrak{n}$ fte $\mathfrak{R} \mathfrak{a} \mathfrak{p}$ itel. \\ Bon bem Berluft Der Rírdiendmter.}

1. Bon ber freimialigen Nieberlegung . $\quad$. . . 242.

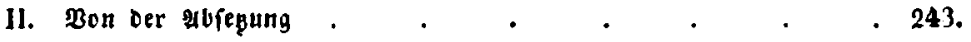

III. Bon Der Berfepung . $\quad . \quad$. . . . . . 244.

\section{ச}

Bon Dem $\mathfrak{B e r m o ̈ g e n ~ b e r ~ R i t d j e . ~}$

Erfte sapitel.

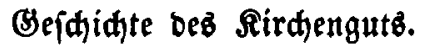

1. 3uftand ber älteren 3eit

II. Entftehung Der Bereficien

III. Entftehung Der Zehnten

IV. Hebergarg Der Sirdengüter in weltlide sönde . . . 248.

V. Fernere Gdiffínle ber Rirdengüter . . . . . . . . 249.

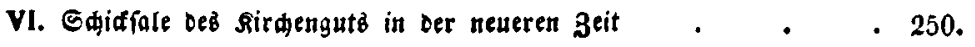

\section{3weiter Rapitel.}

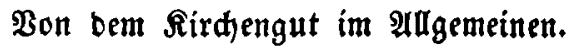

1. Bon Dem Eigentbum am Rirdengut . . . . . 251.

II. Ermerb Der Firdengüter . . . . . . 252.

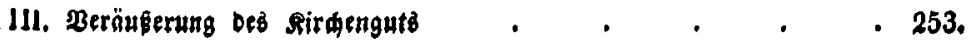


IV. Beftandtheile Dez Rir山engutz.

A) Brunoftüfe, Senten, Eavitalien . . . . . . 254.

B) Primitien, Oblationen, Bebuten . . , . . $25 j$.

v. Borre由te Deß firdengutß . . . . . . 256.

\section{Drittes Rapitel.}

Bon ben $\mathfrak{P}$ frủnben.

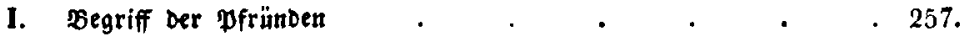

II. Gtiftung der $\mathfrak{P f r u ̈ n d e n ~} \quad . \quad$. $\quad . \quad$. . . . 258.

III. Bern̈noerung einer ptrünoe . . . . . . . . 259.

IV. Redte Der $\mathfrak{P}$ frünoner.
A) 3in थugemeinen
B) Berbältniß̄ in ten Gtiften
- 260 .

v. Beerbung Der Beneficiaten.
A) Relterea Redt
. 262.
B) Bout Gyolienredt
C) Seutiges Redt
- 263.
- 264.
VI. Berwaltung erledigter Pfründen . . . . . . . 265 .

\section{Biertez Iapitel.}

Don ben Rirdjenfabrifen.

I. Siftorifde Einleitung $. \quad . \quad . \quad . \quad . \quad .266$.

IJ. Eintheilung Der Sirdenfabriten . . . . . 267.

111. Bon Den heiligen Saden.
A) Bemeibte Gaden
B) Befegnete Gamen
C) Borredte ber gebeiligten Eaden.
1V. Bon ben gewöhnliden Fabritgütern . . . . . 271 .
V. Bon oer Erbaltung uno berqellung Der Sirgen: und Pfarrgebäube 272 .

\section{Sicbente $B$ ub. \\ Bon bem lirdjlithen $\mathfrak{L e b e n .}$}

Exfter Rapitel.

Bon ben heiligen ḩantolungen im argemeinen.

1. Bon Den Eacraunenten

11. Bon Den Gacramentatien . . . . . . . . 274.

III. Bon Der siturgie.
A) In Der tatbolifden und griediffen sirde
- 275 .
B) In ber proteftantifaen. Sirde
- 276 . 


\section{3weiter Rapitel. \\ Bon bem Eintritt in bie Sirdje.}

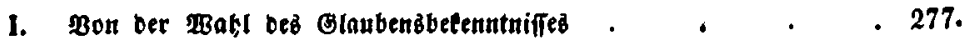

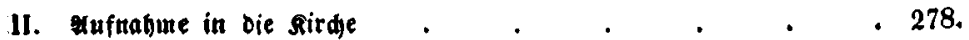

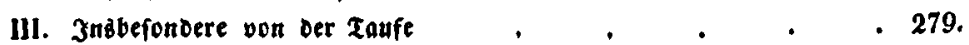

IV. Bon oer firmung , . , , , , . 280.

Dritter Rapltel.

Daz̉ gotteäbienfttidje lébent.

I. Bon ber faeier bes abendmables.
A) Uriprïnglide Form Derfelben
- 281.
B) Bom Empfang oed abenduables . . . . . 282

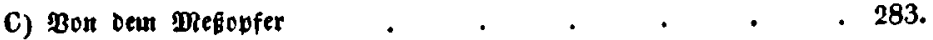
D) Bon ben Mesftipendient uno Defeftiftungen . . , 284 .

II. Bon ber Beidt und Bute.
A) Befentlide Beftandtheile
- 285.
B) ereltere uno beutige Dif́ciplin
- 286 .
C) Gäkęe über Den $2 \mathfrak{U}$ เด
- 287.

III. Bon Dem Bebete.
A) 3in थugemeinen
. 288.

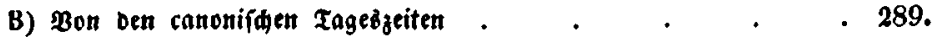
IV. Bon Den Faften
- 290.

V. Diftorifole formen ber Oottedveregrang.
A) Berebrung beiliger perionen
- 291.
B) Berehrung beiliger Beiten .
- 292.
C) Beregrung beiliger Drte $\quad . \quad$. . . . , 293.

\section{Bierteb Rapitel. \\ Bon ber Ehe.}

I. Bon bem wefen ber Ehe $. \quad . \quad . \quad . \quad . \quad$. 294.

II. Befdidte Ded driftliden Eheredtb.
A) Bon Der Gefeggebung in Eheradien
- 295.
B) Bon Der Beriditbarleit it Ebefaden
- 296.

III. Bon ber Eingehung Der Ege.
A) Regeluä̈ßige Erforderniffe.
- 297.
B) Form ber 2bofdliefung.
1) Xettereb Bedit
- 298.

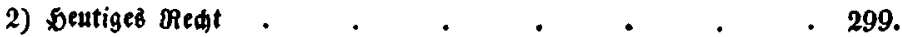

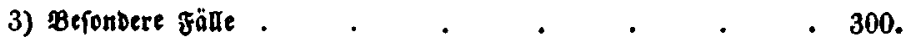
4) Don oer fobe ald Gacrament . . . . . 301. 
IV. Bou betn æBertöbniti.

A) Bebingungen ber Eingehung $\quad . \quad \ldots \quad$. $\quad . \quad 302$.

B) TBirtung ber Berlöbnife . $\quad . \quad$. $\quad . \quad$. $\quad . \quad 303$.

v. Bon ben Ethefinoernifien.

A) Bon bean Redjt Egehinderniffe ju fek̨en . . . 304.

B) Trennende Sinoerniffe.

1) Privatredtlide . $\quad . \quad$ • $\quad$ •

2) Deffentlide.

a) Berfaiedenheit ber Religion . . . . . 306 .

b) Beftehende Berpflidtungen . . . . . 307.

c) Berbreden $\quad$. $\quad . \quad$. $\quad$.

d) Die Berwanttfaft.

ж) Irt Die Berwandtidaft ju berehnen . . . 309

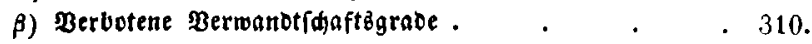

y) Bon Der nadgebildeten Berrondifiaft . . . 311.

e) Die Gdiongerfaft.

a) Birtlide Sdmögerfaft . . . . . 312.

ק) Madgebilbete Edrongeridnft $\quad . \quad$. . . . 313.

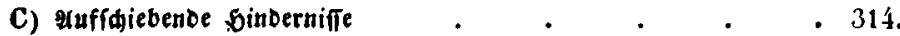

D) Bon ber Diävenfátion bei Ebebinderniffer $\quad . \quad$. 315.

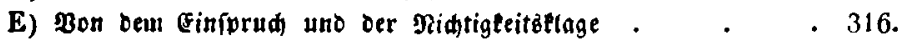

F) Bur Revifion Der Befeggebung über Die Ehefinderniffe - $316 \mathrm{a}$.

VI. Bon ben Birlungen ber हfhe.

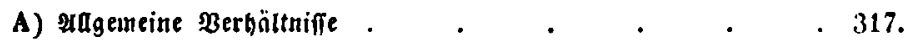

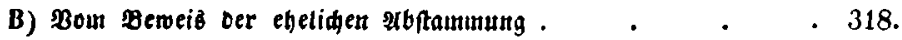

VII. Bon Der Ebefdeibung.

A) Erundebre ber tatbolifden Sirde $\quad$. $\quad$. $\quad$. $\quad 319$.

B) Bon ber Sonberung von Tifh unb Bett . . . 320 .

C) Brieđifdes Rirdenredt . . . . . . . . 321.

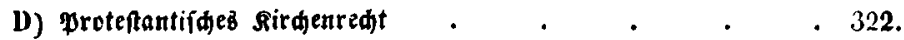

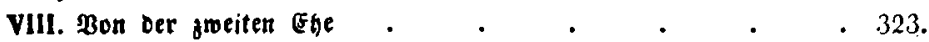

1X. Bon bert gemifder Efen . . . . 324. 324 a.

Fúnftéz Rapitel.

Der dififtlidie $\mathfrak{T o b}$.

1. Bon Der legten Delung . $\quad . \quad$. . . . . 325.

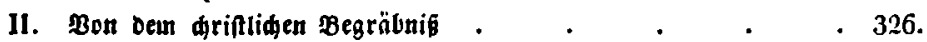

11I. Bom Dienfte ber Berftorbenen . $\quad . \quad$. $\quad . \quad$. 327.

Sedifez $\mathfrak{x a p i t e l}$.

Bon ben befonteren firdiliden Inftalten.

1. Bont Den Boblthätigleitzanftalten.

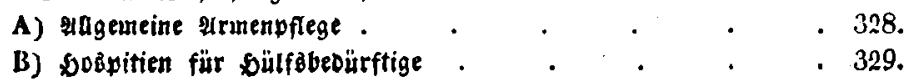


II. Bon Den religiófen Droen.
A) Itagemeine Brandage $\quad . \quad . \quad . \quad, \quad, 330$.
B) Bef由idtlide ueberilgt Der religiöfen Drden . . . 331.

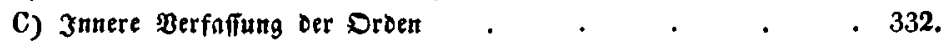
D) Bon Den weibliden Srben $\quad$; $\quad . \quad$. $\quad . \quad$. 333.

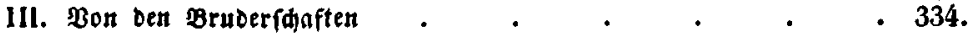
IV. Bon Dert geiftliden Ritterorden $. \quad . \quad . \quad . \quad . \quad 335$.

V. Bont ben Eegrantalten.
A) Elementaridulent
- 336 .
B) Бöbere Sdulen
C) univeritiatatr.

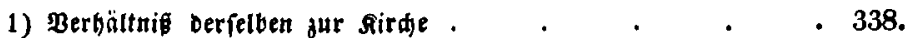
2) Bon Dent theologifmen facultäten $\quad . \quad$. $\quad . \quad$. 339.
3) Bon Den Doctoren ber Theologie . . . . . . 340.

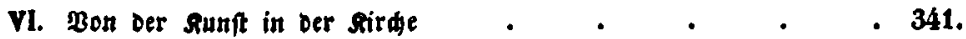

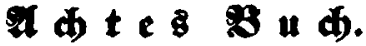

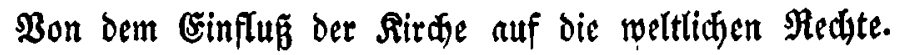

1. Einfuß ber Rirde auf bas Bölterredt . . . . 342.

11. 2uf Das Gtantżredjt . . . . . . . . 343.

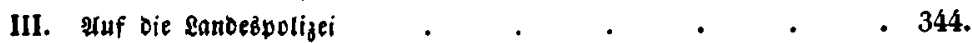

1V. Uนf ১aź Gtrafredt . . . . . . . . . . . 345.

V. 2uf den procep . . . . . . . . . . 346.

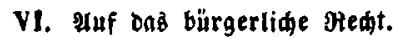

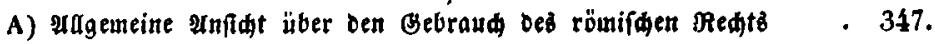
B) Leber ben 3uftano der unfreien . $\quad . \quad \ldots \quad . \quad 348$.
C) Heber Die Teftanente $\quad, \quad, \quad \cdot \quad, \quad, 349$.
D) Ueber Befią, Beriährung uno Berträge . $. \quad . \quad . \quad$. 350 .

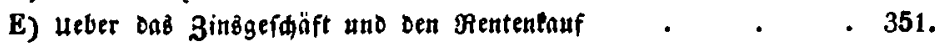
F) Heber Die Berbindlidteit aนz Betäbden . . . . . 352.
G) Heber ben eito.

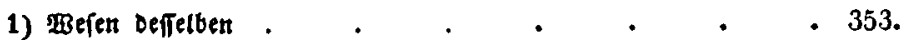
2) Bedingungen und form . $. \quad . \quad . \quad . \quad . \quad . \quad 354$.

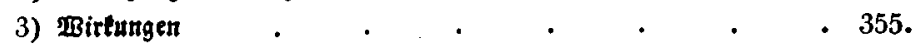

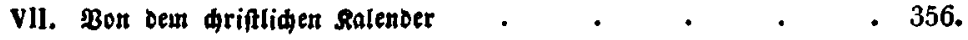

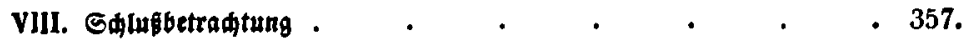




\section{$\mathbf{a} \mathbf{n} \mathbf{b} \mathfrak{a} \mathbf{n}$.}

Bayetr.

Geite

Concorbat vom 5. Jutti 1817 . . . . . . 695 .

Religionbebitt vom 26. Mai 1818 . . . . . . 700.

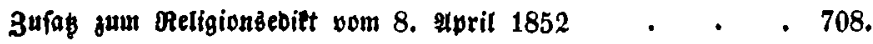

Şannover.

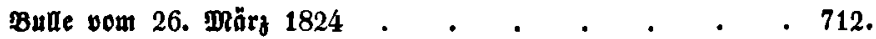

\section{Oherrheinifde Sitrdenproving.}

Bulle vom 16. 2uguft 1821, . . . . . , 719.

Bulle vom 11. Alyril 1827 . . . . . . . . . 727.

Eoict vom 30. $\mathfrak{3 a n u a r} 1830$. $\quad . \quad . \quad . \quad . \quad . \quad . \quad .730$.

2poftol. Breve voun 30. 3uni $1830 \quad$. $\quad . \quad . \quad . \quad . \quad 733$.

Eoict vom 1. Mär 1853 . . . . . . . . . 735.

Defterreid).

saiferl. Beroronung vop 18. 2pril 1850 . . . . 736.

Raiferl. Berordaung vom 23. 2tpril $1850 \quad$ • $\quad . \quad$. $\quad 738$.

Raiferl. Watent vom 31. December $1851 \quad$. . . . 738.

Goncorbat vom 18. auguft 1855 . . . . . . . 739:

Senifert. Ertlörung vom 18. Augutt 1855 . . . . 745 .

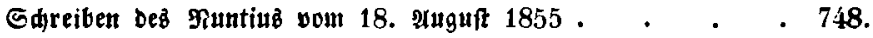

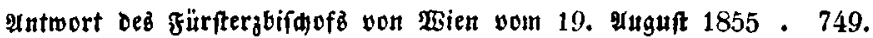

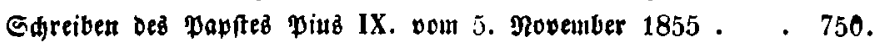

Preußen.

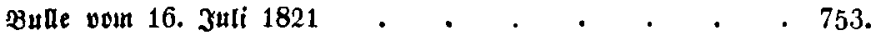

Rabinetzorore vom 23. 2tuguft $1821 \quad$. . . . . . 767.

Berfafiung vou 31. 3anuar 1850 . . . . . . 768.

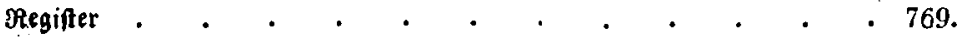

